PROCEEDINGS OF THE

AMERICAN MATHEMATICAL SOCIETY

Volume 134, Number 5, Pages 1249-1256

S 0002-9939(05)08425-X

Article electronically published on December 14, 2005

\title{
ON QUASI-COMPLETE INTERSECTIONS OF CODIMENSION 2
}

\author{
YOUNGOOK CHOI
}

(Communicated by Bernd Ulrich)

\begin{abstract}
In this paper, we prove that if $X \subset \mathbb{P}^{n}, n \geq 4$, is a locally complete intersection of pure codimension 2 and defined scheme-theoretically by three hypersurfaces of degrees $d_{1} \geq d_{2} \geq d_{3}$, then $H^{1}\left(\mathbb{P}^{n}, \mathcal{I}_{X}(j)\right)=0$ for $j<d_{3}$ using liaison theory and the Arapura vanishing theorem for singular varieties. As a corollary, a smooth threefold $X \subset \mathbb{P}^{5}$ is projectively normal if $X$ is defined by three quintic hypersurfaces.
\end{abstract}

\section{INTRODUCTION}

Let $X$ be a nondegenerate projective variety of codimension $e$ and degree $d$ in $\mathbb{P}^{n}$, defined over a complex number field $\mathbb{C}$. $X$ is said to be the scheme-theoretic intersection of $k+1$ homogeneous equations $f_{1}, f_{2}, \ldots, f_{k+1}$ if the saturation of an ideal $J=\left(f_{1}, \ldots, f_{k+1}\right)$ is equal to the homogeneous ideal $I_{X}=\bigoplus_{j \in \mathbb{Z}} H^{0}\left(\mathbb{P}^{N}, \mathcal{I}_{X}(j)\right)$ of $X . X$ is called a quasi-complete intersection (qci for short) if $X$ is a schemetheoretic intersection of $e(=\operatorname{codim} X)+1$ equations. In their paper $([3]$, Proposition 1 ), Beorchia and Ellia showed that if $X$ is a smooth codimension 2 qci, which is not a complete intersection, of three hypersurfaces of degrees $d_{1}, d_{2}, d_{3}$, then the integers $d_{i}$ are determined by $X$; that is, if $X$ is a qci of three other hypersurfaces of degrees $d_{1}{ }^{\prime}, d_{2}{ }^{\prime}, d_{3}{ }^{\prime}$, then $d_{i}=d_{i}{ }^{\prime}$ for $i=1,2,3$ up to order. This result is generalized by Bresinsky, Schenzel, and Stückrad ([5]) for irreducible and reduced (not necessarily smooth) qci's of codimension 2 which are not complete intersections.

Definition 1.1. A variety $X \subset \mathbb{P}^{n}$ is called a qci of type $\left(d_{1}, d_{2}, d_{3}\right)$ if $X$ is a codimension two, quasi-complete intersection of three hypersurfaces of degrees $d_{1}, d_{2}, d_{3}$ with $d_{1} \geq d_{2} \geq d_{3}$ and general 3 -dimensional plane sections of $X$ are smooth. A variety whose homogeneous ideal $I_{X}$ is generated by $e+1$ equations is called an almost complete intersection.

This paper is concentrated on the cohomological properties of quasi-complete intersections of codimension two. $X \subset \mathbb{P}^{n}$ is said to be $t$-normal if the cohomology group $H^{1}\left(\mathbb{P}^{n}, \mathcal{I}_{X}(t)\right)=0$. By the Serre vanishing theorem, $X$ is $t$-normal for all $t \gg 0$, and it would be very interesting to find an explicit lower bound $B(d, e)$ in terms of its degree $d$ and codimension $e$ such that $X$ is $t$-normal for all $t \geq B(d, e)$. For example, S. Kwak ([13]) showed that any smooth threefold $X \subset \mathbb{P}^{5}$ of degree

Received by the editors September 10, 2004.

2000 Mathematics Subject Classification. Primary 14M07, 14N05, 14M06.

Key words and phrases. Quasi-complete intersections, liaison, normality, defining equations.

The author was supported in part by KRF (grant No. KRF-2002-070-C00003).

(C)2005 American Mathematical Society Reverts to public domain 28 years from publication 
$d$ is $t$-normal for all $t \geq d-4$ and that this bound is sharp as the Palatini scroll of degree 7 shows. In their paper (4, Proposition 1), Bertram-Ein-Lazarsfeld gave an interesting bound about $t$-normality in terms of degrees of defining equations of $X$ : Let $X \subset \mathbb{P}^{n}$ be the scheme-theoretic intersection of $m$ equations $f_{1}, f_{2}, \ldots, f_{m}$ with degrees $d_{1} \geq d_{2} \geq \cdots \geq d_{m}, m \geq e=\operatorname{codim}(X)$. Then we have

$$
H^{i}\left(\mathbb{P}^{n}, \mathcal{I}_{X}(j)\right)=0, i \geq 1, j \geq d_{1}+d_{2}+\cdots+d_{e}-n .
$$

For a smooth qci of type $\left(d_{1}, d_{2}, d_{3}\right)$, we get the following theorem.

Theorem 1.2. Let $X$ be a smooth codimension two subvariety in $\mathbb{P}^{n}, n=4,5$. If $X$ is a quasi-complete intersection of type $\left(d_{1}, d_{2}, d_{3}\right)$ with a sheaf of ideals $\mathcal{I}_{X}$, then $H^{i}\left(\mathcal{I}_{X}(k)\right)=0$ for $k \geq d_{1}+d_{2}+d_{3}-2 n+1$ and $i \geq 1$.

On the other hand, for projective varieties of small codimension, it has also been focused on the vanishing of cohomology groups $H^{i}\left(\mathbb{P}^{n}, \mathcal{I}_{X}(j)\right)$ for a small positive integer $j$. For example, a famous theorem of Zak's tells us that any smooth threefold is linearly normal, i.e. $H^{1}\left(\mathbb{P}^{5}, \mathcal{I}_{X}(1)\right)=0$. In this sense, it is quite useful to know the following conjecture due to Peskine and Zak: Let $X$ be a nondegenerate, not necessarily smooth, projective variety of codimension $e$ in $\mathbb{P}^{n}$ :

(1) $H^{i}\left(\mathbb{P}^{n}, \mathcal{I}_{X}(j)\right)=0$ for $i \geq 1, j \geq 0, i+j<\frac{\operatorname{dim}(X)}{e-1}$.

(2) For $i \geq 1, j \geq 0, i+j=\frac{\operatorname{dim}(X)}{e-1}$, it is possible to describe all varieties for which $H^{i}\left(\mathbb{P}^{n}, \mathcal{I}_{X}(j)\right) \neq 0$.

Now we would like to consider this conjecture from the point of defining equations of codimension two qci subvarieties. Note that all complete intersections and almost complete intersections of codimension two are projectively Cohen-Macaulay. For quasi-complete intersections, we can prove the following theorem using liaison theory and the Arapura vanishing theorem.

Theorem 1.3. Let $X \subset \mathbb{P}^{n}, n \geq 4$, be a reduced locally complete intersection of codimension 2 . If $X$ is a quasi-complete intersection of type $\left(d_{1}, d_{2}, d_{3}\right)$, then $H^{1}\left(\mathbb{P}^{n}, \mathcal{I}_{X}(k)\right)=0$ for $k<d_{3}$. Furthermore, if $\operatorname{dim}(X)=3$ and the singular locus of $X$ is finite, then $H^{1}\left(\mathbb{P}^{n}, \mathcal{I}_{X}(k)\right)=0$ for $k \leq d_{3}$ and $H^{2}\left(\mathbb{P}^{n}, \mathcal{I}_{X}(k)\right)=0$ for $k<d_{3}$.

A corollary of these two theorems is:

Corollary 1.4. If $X$ is a smooth threefold in $\mathbb{P}^{5}$ that is cut out scheme-theoretically by three equations of degrees $d_{1} \geq d_{2} \geq d_{3}$ with $d_{1}+d_{2} \leq 10$, then $X$ is projectively normal, and if $d_{1}+d_{2} \leq 9$, then $X$ is projectively Cohen-Macaulay.

In Section 2, we will review some basic backgrounds including the Peskine-Szpiro Theorem ([16, Proposition 4.1) and the Gherardelli Linkage Theorem ([10, Theorem 2.5) needed in this paper. In Section 3, we will prove the above theorems and give some corollaries and examples.

\section{Preliminaries}

The following two theorems are generalizations of the classical Kodaira vanishing theorem for ample vector bundles and for singular subvarieties. 
Theorem 2.1. Let $\mathcal{E}$ be a vector bundle of rank $r$ on $\mathbb{P}^{n}$, and let $\mathcal{L}$ be an ample line bundle. Assume that $\mathcal{E}$ is generated by its global sections. Then

$$
h^{q}\left(\mathbb{P}^{n}, \bigwedge^{t} \mathcal{E} \otimes \mathcal{L} \otimes K_{\mathbb{P}^{n}}\right)=0 \text { for } q>r-t .
$$

Proof. See [8], page 54 .

Theorem 2.2. Let $Y$ be a projective variety, and let $\mathcal{L}$ be an ample line bundle on $Y$. Fix $k \geq 1$. Assume that $k<\operatorname{codim}(\operatorname{Sing}(Y))$ and that $Y$ is $S_{k+1}$. Then $H^{k}\left(Y, \mathcal{L}^{-1}\right)=0$. (If $Y$ is smooth, we define $\operatorname{codim}(\operatorname{Sing}(Y))=\operatorname{dim}(Y)$.)

Proof. See [1, Proposition 1.1.

Theorem 2.3 (Gherardelli Linkage Theorem). Let $X \subset \mathbb{P}^{n}$ be a Cohen-Macaulay subvariety of pure codimension 2 and $Z$ a complete intersection of two hypersurfaces $S_{1}$ and $S_{2}$. Assume that $Z$ has pure codimension 2. Then $X \subset Z$ is subcanonical, i.e. $K_{X}=\mathcal{O}(k)$ for some $k \in \mathbb{Z}$ if and only if its residual scheme $Y \subset Z$ of $X$ is scheme-theoretically defined by the intersection of $S_{1}$ and $S_{2}$ with a third hypersurface $S_{3}$. In addition, if $d_{i}=\operatorname{deg}\left(S_{i}\right)$ for $i=1,2,3$, then

$$
K_{X}=\mathcal{O}_{X}\left(d_{1}+d_{2}-d_{3}-n-1\right) .
$$

Proof. See [10, Theorem 2.5.

Theorem 2.4 (Peskine-Szpiro Theorem). Let $X$ be a closed subscheme without embedded components, that is a generically complete intersection of codimension 2 in $\mathbb{P}=\mathbb{P}_{k}^{l}$, defined by a graded ideal $I$ in $k\left[X_{0}, \ldots, X_{l}\right]$. Let $U_{1}$ be an open set consisting of points in $X$ where $X$ is complete intersection in $\mathbb{P}$ and $U_{2} \subset X$ consisting of regular points in $X$.

Then there exist homogeneous equations $\alpha_{1}, \alpha_{2} \in I$ defining two hypersurfaces $S_{\alpha_{1}}$ and $S_{\alpha_{2}}$ which intersect properly such that $S_{\alpha_{1}} \cap S_{\alpha_{2}}=X \cup Y$, where

1) $X$ and $Y$ have no common components.

2) $Y \cap U_{1}$ is a locally complete intersection.

3) $Y$ is a complete intersection at all points of codim $\leq 3$ in $Y \cap U_{1}$.

4) $Y-X \cap Y$ is non-singular.

5) $Y \cap U_{2}$ is non-singular in codimension 2 and

$$
\operatorname{codim}\left(Y \cap\left(U_{1}-U_{2}\right),\left(U_{1}-U_{2}\right)\right) \geq 1 .
$$

6) $Y$ is non-singular at all points $x \in Y \cap U_{2}$ of codimension $\leq 3$ in $Y$.

Moreover, if char $k=0$, one can take the elements $\alpha_{i}$ of degree $d_{i}$ provided that

$$
d_{1} \geq \inf \left\{s: I_{s} k\left[X_{0}, X_{1}, \ldots, X_{l}\right] \text { defines } X\right\},
$$$$
d_{2} \geq \inf \left\{t: I_{t} k\left[X_{0}, X_{1}, \ldots, X_{l}\right] / \alpha_{1} \text { defines } X \text { in } k\left[X_{0}, X_{1}, \ldots, X_{l}\right] / \alpha_{1}\right\} \text {. }
$$

Proof. See [16, Proposition 4.1.

We will apply Theorem 2.4 for a locally complete intersection $X$ of codimension two in $\mathbb{P}^{n}$.

Remark 2.5. If $X$ is a qci of type $\left(d_{1}, d_{2}, d_{3}\right)$, then by Theorem 2.4 one can find two hypersurfaces $S_{\alpha_{1}}$ and $S_{\alpha_{2}}$ of degrees $d_{1}$ and $d_{2}$ satisfying the properties of Theorem 2.4 in such a way that $\left\{\alpha_{1}, \alpha_{2}\right\}$ belong to a set of minimal generators of $I_{X}$. By Theorem 2.3. the residual $Y$ of $X$ (in the complete intersection of $S_{\alpha_{1}}$ and $S_{\alpha_{2}}$ ) is subcanonical and holds the properties of Theorem 2.4. 
Theorem 2.6. Let $\mathcal{E}$ be a locally free sheaf on $\mathbb{P}^{n}$. If $\operatorname{rank}(\mathcal{E})<n$ and $n$ is even, then $\mathcal{E}$ splits if and only if $H_{*}^{i}(\mathcal{E})=0$ for $1<i<n-1$. If $\operatorname{rank}(\mathcal{E})<n-1$ and $n$ is odd, then $\mathcal{E}$ splits if and only if $H_{*}^{i}(\mathcal{E})=0$ for $1<i<n-1$.

Proof. See [14, Theorem 1.

Corollary 2.7. Let $X$ be a Cohen-Macaulay surface in $\mathbb{P}^{4}$ defined by three hypersurfaces. Then $X$ is projectively normal if and only if $X$ is projectively CohenMacaulay.

Proof. If $X$ is defined by three hypersurfaces of degrees $d_{1}, d_{2}, d_{3}$, then $\mathcal{I}_{X}$ has the following resolution:

$$
0 \rightarrow \mathcal{E} \rightarrow \mathcal{O}_{\mathbb{P}^{4}}\left(-d_{1}\right) \oplus \mathcal{O}_{\mathbb{P}^{4}}\left(-d_{2}\right) \oplus \mathcal{O}_{\mathbb{P}^{4}}\left(-d_{3}\right) \stackrel{\varphi}{\rightarrow} \mathcal{I}_{X} \rightarrow 0,
$$

where $\mathcal{E}$ is a kernel of a canonical map $\varphi$. Since $X$ is Cohen-Macaulay, $\mathcal{E}$ is a vector bundle of rank two on $\mathbb{P}^{4}$. If $X$ is projectively normal, then $H^{1}\left(\mathbb{P}^{4}, \mathcal{I}(k)\right)=$ $H^{2}\left(\mathbb{P}^{4}, \mathcal{E}(k)\right)=0$ for all $k \in \mathbb{Z}$. By Theorem 2.6, $\mathcal{E}$ splits into line bundles, which implies that $X$ is projectively Cohen-Macaulay.

\section{Main RESUlts AND APPLications}

As Mumford defined, for a coherent sheaf $\mathcal{F}$ on $\mathbb{P}^{n}$ it is said to be $m$-regular if $H^{i}\left(\mathbb{P}^{n}, \mathcal{F}(m-i)\right)=0$ for all $i>0$, and the regularity of $\mathcal{F}$ is defined by the formula

$$
\operatorname{reg} \mathcal{F}=\min \{m \in \mathbb{Z}: \mathcal{F} \text { is } m \text {-regular }\} \text {. }
$$

Lemma 3.1. Let $X$ be a smooth non-degenerate subvariety of codimension two in $\mathbb{P}^{n}, n=4,5$. Assume that $X$ is defined by $(k+1)$ hypersurfaces of degrees $d_{1}, \ldots, d_{k+1}$, i.e. there is a locally free resolution

$$
0 \rightarrow \mathcal{E} \rightarrow \bigoplus_{i=1}^{k+1} \mathcal{O}_{\mathbb{P}^{n}}\left(-d_{i}\right) \rightarrow \mathcal{I}_{X} \rightarrow 0
$$

Then

(a) If $\operatorname{dim} X=2$, then $\operatorname{reg}\left(\mathcal{E}^{*}\right) \leq-3$.

(b) If $\operatorname{dim} X=3$ and $X$ is not in a cubic hypersurface, then $\operatorname{reg}\left(\mathcal{E}^{*}\right) \leq-4$.

Proof. (a) First note that $\mathcal{E}$ is a locally free sheaf of rank $k$ because $X$ is smooth of codimension two. By definition, $\mathcal{E}^{*}$ is $(-3)$-regular if and only if

$$
H^{i}\left(\mathbb{P}^{4}, \mathcal{E}^{*}(-3-i)\right)=0
$$

for all $i \geq 1$. By Serre's duality, this is equivalent to $H^{j}\left(\mathbb{P}^{4}, \mathcal{E}(2-j)\right)=0$ for $0 \leq j \leq 3$. From an exact sequence (10), when $j=3$, we have by the Kodaira vanishing theorem

$$
H^{3}\left(\mathbb{P}^{4}, \mathcal{E}(-1)\right)=H^{2}\left(\mathcal{I}_{X}(-1)\right)=H^{1}\left(\mathcal{O}_{X}(-1)\right)=0 .
$$

Note also that for $j=0,1,2$

$$
\begin{gathered}
H^{2}\left(\mathbb{P}^{4}, \mathcal{E}\right)=H^{1}\left(\mathcal{I}_{X}\right)=0(X \text { is smooth }), \\
H^{1}\left(\mathbb{P}^{4}, \mathcal{E}(1)\right)=0(\mathrm{X} \text { is non-degenerate }), \\
H^{0}\left(\mathbb{P}^{4}, \mathcal{E}(2)\right)=0 .
\end{gathered}
$$

Note that the last vanishing comes from the exact sequence (1) and the nondegeneracy of $X$.

(b) See [6, Lemma 3.7 . 
Note that by Faltings theorem $([9, \S 4)$, all smooth quasi-complete intersections $X \subset \mathbb{P}^{n}$ of codimension 2 are complete intersections if $n \geq 6$.

Theorem 3.2. Let $X$ be a smooth codimension two subvariety in $\mathbb{P}^{n}, n=4,5$. If $X$ is a quasi-complete intersection of type $\left(d_{1}, d_{2}, d_{3}\right)$ with an ideal sheaf $\mathcal{I}_{X}$, then:

(a) $H^{i}\left(\mathbb{P}^{n}, \mathcal{I}_{X}(k)\right)=0$ for $k \leq \operatorname{dim} X$ and $1 \leq i<\operatorname{dim} X$.

(b) $H^{i}\left(\mathbb{P}^{n}, \mathcal{I}_{X}(k)\right)=0$ for $k \geq d_{1}+d_{2}+d_{3}-2 n+1$ and $i \geq 1$.

Proof. (a) Assume that $\operatorname{dim} X=3$. If $X$ is contained in a cubic hypersurface, then it is projectively Cohen-Macaulay (7], Proposition 5.1), so we assume that $X$ is not contained in a cubic hypersurface. By Lemma 3.1, $\mathcal{E}^{*}$ is $(-4)$-regular, so $\mathcal{E}^{*}(-4)$ is generated by its global sections. Applying Theorem 2.1. one can get

$$
H^{i}\left(\mathbb{P}^{5}, K_{\mathbb{P}^{5}} \otimes \mathcal{E}^{*}(-4) \otimes \mathcal{O}(k)\right)=0 \text { for } k \geq 1, i>1 .
$$

By Serre duality, $H^{i}\left(\mathbb{P}^{5}, \mathcal{E}(4-k)\right)=0$ for $k \geq 1$ and $0 \leq i \leq 3$. This gives $H^{i}\left(\mathcal{I}_{X}(k)\right)=0$ for $k \leq 3$ and $1 \leq i<3$. For $\operatorname{dim} X=2$, by Lemma 3.1, $\mathcal{E}^{*}$ is $(-3)$-regular and, in the same way, we get $H^{1}\left(\mathcal{I}_{X}(k)\right)=0$ for $k \leq 2$.

(b) Assume that $\operatorname{dim} X=3$. Note that $\mathcal{E}$ in the exact sequence (11) is locally free of rank 2 and $c_{1}(\mathcal{E})=-\left(d_{1}+d_{2}+d_{3}\right)$. So $\mathcal{E}^{*}(-4)=\mathcal{E}\left(d_{1}+d_{2}+d_{3}-4\right)$, and for $k \geq 1$ and $i>1$,

$$
H^{i}\left(\mathbb{P}^{5}, \mathcal{E}\left(d_{1}+d_{2}+d_{3}-10+k\right)\right)=H^{i}\left(\mathbb{P}^{5}, K_{\mathbb{P}^{5}} \otimes \mathcal{E}^{*}(-4) \otimes \mathcal{O}(k)\right)=0 .
$$

This implies $H^{i}\left(\mathcal{I}_{X}(k)\right)=0$ for $k \geq d_{1}+d_{2}+d_{3}-9$ and $i \geq 1$. Similar proof goes for $\operatorname{dim} X=2$.

This method can be applied to a smooth threefold in $\mathbb{P}^{5}$ which is cut out by four equations.

Theorem 3.3. Let $X$ be a smooth threefold in $\mathbb{P}^{5}$. If $X$ is cut out schemetheoretically by four hypersurfaces of degrees $d_{1}, d_{2}, d_{3}, d_{4}$, then:

(a) $H^{1}\left(\mathbb{P}^{5}, \mathcal{I}_{X}(k)\right)=0$ for $k \leq 3$.

(b) $H^{i}\left(\mathbb{P}^{5}, \mathcal{I}_{X}(k)\right)=0$ for $k \geq d_{1}+d_{2}+d_{3}+d_{4}-13$ and $i \geq 1$.

Proof. (a) The proof is similar to the proof of Theorem 3.2(a). 2.1 .

(b) By Lemma 3.1, $\mathcal{E}^{*}(-4)$ is generated by its global sections, and by Theorem

$$
H^{i}\left(\mathbb{P}^{5}, K_{\mathbb{P}^{5}} \otimes \bigwedge^{2} \mathcal{E}^{*}(-4) \otimes \mathcal{O}(k)\right)=0 \text { for } k \geq 1, i>1 .
$$

Since $X$ is cut out by 4 hypersurfaces, $\mathcal{E}^{*}$ is locally free of rank 3 . So $\bigwedge^{2} \mathcal{E}^{*}(-4)=$ $\mathcal{E}\left(d_{1}+d_{2}+d_{3}+d_{4}-8\right)$ and $H^{i}\left(\mathbb{P}^{5}, \mathcal{E}\left(d_{1}+d_{2}+d_{3}+d_{4}-14+k\right)\right)=0$ for $i>1$ and $k \geq 1$. Therefore, $H^{i}\left(\mathcal{I}_{X}(k)\right)=0$ for $k \geq d_{1}+d_{2}+d_{3}+d_{4}-13$ and $i \geq 1$.

Corollary 3.4. In the situation of Theorem 3.3 above, if $d_{1}+d_{2}+d_{3}+d_{4} \leq 17$, then $X$ is projectively normal.

Theorem 3.5. Let $X$ be a smooth surface in $\mathbb{P}^{4}$. If $X$ is cut out scheme-theoretically by four hypersurfaces of degrees $d_{1}, d_{2}, d_{3}, d_{4}$, then $H^{i}\left(\mathcal{I}_{X}(k)\right)=0$ for $k \geq d_{1}+$ $d_{2}+d_{3}+d_{4}-10$, and $i \geq 1$.

Proof. The proof is similar to the proof of Theorem $3.3(\mathrm{~b})$. 
Remark 3.6. Assume that $X$ is a projected Veronese surface in $\mathbb{P}^{4}$. Clearly, $X$ is not a linearly normal, i.e. $H^{1}\left(\mathcal{I}_{X}(1)\right) \neq 0$. By the way, $X$ has the following exact sequence:

$$
0 \rightarrow \mathcal{F}^{*}(-4) \rightarrow \mathcal{O}_{\mathbb{P}^{4}}(-3)^{\oplus 4} \rightarrow \mathcal{I}_{X} \rightarrow 0,
$$

where $\mathcal{F}$ is the Tango bundle on $\mathbb{P}^{4}$. So, $X$ is cut out by four cubic hypersurfaces. This shows that Theorem 3.5] is sharp.

From now on, we do not assume that $X$ is smooth. As usual, liaison theory is a tool for studying a qci of codimension two.

Theorem 3.7. Let $X \subset \mathbb{P}^{n}, n \geq 4$, be a Cohen-Macaulay and quasi-complete intersection of type $\left(d_{1}, d_{2}, d_{3}\right)$ and $Y$ a residual of $X$ in the complete intersection of hypersurfaces $S_{1}$ and $S_{2}$ of degrees $d_{1}$ and $d_{2}$, i.e. $X \cup Y=S_{1} \cap S_{2}$. If $\operatorname{codim}(\operatorname{Sing}(Y))=t$ for some $t \in \mathbb{Z}$, then:

(a) $H^{i}\left(\mathbb{P}^{n}, \mathcal{I}_{X}(k)\right)=0$ for $0 \leq i<t$ and $k<d_{3}$, and

(b) $H^{i}\left(\mathbb{P}^{n}, \mathcal{I}_{X}(k)\right)=0$ for $i>n-2-t, k \geq d_{1}+d_{2}-n$.

Proof. (a) First of all, since $d_{3}$ is a minimal degree of a hypersurface containing $X$ ([11]), $H^{0}\left(\mathcal{I}_{X}(k)\right)=0$ for $k<d_{3}$. Since $X$ is a qci of codimension 2, by Theorem 2.3. $Y$ is a subcanonical with $K_{Y}=\mathcal{O}_{Y}\left(d_{1}+d_{2}-d_{3}-n-1\right)$. Since $X \cup Y=S_{1} \cap S_{2}$, the liaison theory gives the following exact sequence ([15], page 117):

$$
0 \rightarrow \mathcal{I}_{X \cup Y} \rightarrow \mathcal{I}_{X} \rightarrow K_{Y}\left(n+1-d_{1}-d_{2}\right) \rightarrow 0 .
$$

Therefore we have

$$
0 \rightarrow \mathcal{I}_{X \cup Y}\left(d_{3}\right) \rightarrow \mathcal{I}_{X}\left(d_{3}\right) \rightarrow \mathcal{O}_{Y} \rightarrow 0 .
$$

As $X \cup Y$ is a complete intersection, $H^{i}\left(\mathcal{I}_{X \cup Y}(k)\right)=0$ for $1 \leq i \leq n-2$ and for all $k \in \mathbb{Z}$. Note that the residual $Y$ is Cohen-Macaulay if $X$ is Cohen-Macaulay ([10, Proposition 2.1). So by Theorem 2.2. we have $H^{i}\left(\mathcal{O}_{Y}(k)\right)=0$ for $0 \leq i<t$ and $k<0$. Therefore $H^{i}\left(\mathcal{I}_{X}(k)\right)=0$ for $k<d_{3}$ and $0 \leq i<t$.

(b) By Serre duality and Theorem 2.2. $H^{i}\left(K_{Y}(k)\right)=0$ for $i>n-2-t$ and $k \geq 1$. Since $X \cup Y$ is a complete intersection of degrees $d_{1}$ and $d_{2}, H^{i}\left(\mathcal{I}_{X \cup Y}(k)\right)=0$ for $i \geq 1$ and for all $k \geq d_{1}+d_{2}-n$. So we get, from the exact sequence (3), $H^{i}\left(\mathbb{P}^{n}, \mathcal{I}_{X}(k)\right)=0$ for $i>n-2-t, k \geq d_{1}+d_{2}-n$.

In their paper (4]), Bertram-Ein-Lazarsfeld show that if $X$ is a smooth qci of type $\left(d_{1}, d_{2}, d_{3}\right)$, then $H^{i}\left(\mathbb{P}^{n}, \mathcal{I}_{X}(k)\right)=0$ for $k \geq d_{1}+d_{2}-n$ and $i \geq 1$. So if $Y$ is smooth, the Kodaira vanishing theorem holds for $Y$, i.e. $H^{i}\left(\mathbb{P}^{n}, K_{Y}(k)\right)=0$ for $k \geq 1, i \geq 1$. So one may consider (b) as a generalization of the Bertram-EinLazarsfeld theorem for singular varieties.

If $X$ is a locally complete intersection, then one can control singular locus $\operatorname{Sing}(Y)$ of residual $Y$ of $X$ via the Peskine-Szpiro theorem (Theorem 2.4). Using this theorem, one can state the theorem without mentioning a residual $Y$ of $X$.

Theorem 3.8. Let $X \subset \mathbb{P}^{n}, n \geq 4$, be a reduced locally complete intersection of codimension 2 . If $X$ is a quasi-complete intersection of type $\left(d_{1}, d_{2}, d_{3}\right)$, then:

(a) $H^{1}\left(\mathbb{P}^{n}, \mathcal{I}_{X}(k)\right)=0$ for $k<d_{3}$ and

(b) $H^{i}\left(\mathbb{P}^{n}, \mathcal{I}_{X}(k)\right)=0$ for $i>n-4, k \geq d_{1}+d_{2}-n$. 
If $\operatorname{dim}(X)=3$ and the singular locus of $X$ is finite, then

(c) $H^{1}\left(\mathbb{P}^{5}, \mathcal{I}_{X}(k)\right)=0$ for $k \leq d_{3}$ and

(d) $H^{2}\left(\mathbb{P}^{5}, \mathcal{I}_{X}(k)\right)=0$ for $k<d_{3}$ and

(e) $H^{i}\left(\mathbb{P}^{5}, \mathcal{I}_{X}(k)\right)=0$ for $i \geq 1, k \geq d_{1}+d_{2}-n$.

Proof. (a) As in Remark 2.5, one can find two hypersurfaces $S_{1}$ and $S_{2}$ of degree $d_{1}, d_{2}$ containing $X$ such that the residual $Y$ of $X$ (in the complete intersection of $S_{1}$ and $S_{2}$ ) is subcanonical and holds the properties of Theorem 2.4. Properties (4) and (6) in Theorem 2.4 say $Y$ is regular in codimension 3 at all points in $Y-\operatorname{Sing}(X) \cap Y$. From property $(5), \operatorname{codim}(\operatorname{Sing}(X) \cap Y, \operatorname{Sing}(X)) \geq 1$. Since $X$ is reduced, $\operatorname{codim}(\operatorname{Sing}(X), X) \geq 1$. So we can conclude that $\operatorname{codim}(\operatorname{Sing}(Y), Y) \geq 2$. By Theorem 2.2, $H^{1}\left(\mathcal{O}_{Y}(k)\right)=0$ for $k<0$. From the above exact sequence (3), $H^{1}\left(\mathbb{P}^{n}, \mathcal{I}_{X}(k)\right)=0$ for $k<d_{3}$.

(b) As in the proof of (a), one can find a residual $Y$ of $X$ satisfying $H^{1}\left(\mathcal{O}_{Y}(k)\right)=$ 0 for $k<0$. Using Serre duality and an exact sequence (3), $H^{i}\left(\mathcal{I}_{X}(k)\right)=0$ for $i>n-4, k \geq d_{1}+d_{2}-n$.

For the second statement, if $\operatorname{dim}(X)=3$ and $X$ is a local complete intersection with a zero-dimensional singular locus, then by Theorem 2.4 one can find a smooth residual $Y$ of $X$ which has no component in common with $X$. So, by Theorem 2.2. $H^{i}\left(\mathcal{O}_{Y}(k)\right)=0$ for $k<0$ and $i=1,2$. Using Serre duality and an exact sequence (31), one gets (d) and (e). For (c), since $\operatorname{dim} Y=3$ and $Y$ is smooth, by Barth's theorem, $H^{1}\left(\mathcal{O}_{Y}\right)=0$. So we get $H^{1}\left(\mathbb{P}^{5}, \mathcal{I}_{X}(k)\right)=0$ for $k \leq d_{3}$.

Corollary 3.9. If $X$ is a smooth threefold in $\mathbb{P}^{5}$ cut out scheme-theoretically by three equations of degrees $d_{1} \geq d_{2} \geq d_{3}$ with $d_{1}+d_{2} \leq 10$, then $X$ is projectively normal, and if $d_{1}+d_{2} \leq 9$, then $X$ is projectively Cohen-Macaulay.

Proof. By Theorem 3.8, $H^{i}\left(\mathbb{P}^{5}, \mathcal{I}_{X}(k)\right)=0$ for $k<d_{3}, i=1,2$, and by Theorem 3.2. $H^{i}\left(\mathbb{P}^{5}, \mathcal{I}_{X}(k)\right)=0$ for $k \geq d_{1}+d_{2}+d_{3}-9$ and $i \geq 1$. So if $d_{1}+d_{2} \leq 9$, then $H^{i}\left(\mathbb{P}^{5}, \mathcal{I}_{X}(k)\right)=0$ for all $k \in \mathbb{Z}$ and $i=1,2$. Then $H^{i}\left(\mathbb{P}^{5}, \mathcal{E}(k)\right)=0$ for all $k \in \mathbb{Z}$ and $i=2,3$, where $\mathcal{E}$ is is a rank two vector bundle in the exact sequence (11) of Lemma 3.1. By Theorem 2.6, $\mathcal{E}$ splits into line bundles. So $X$ is projectively Cohen-Macaulay.

Since $H^{1}\left(\mathbb{P}^{5}, \mathcal{I}_{X}(k)\right)=0$ for $k \leq d_{3}$ by Theorem 3.8 (c), it is clear that if $d_{1}+d_{2} \leq 10$, then $X$ is projectively normal, i.e. $H^{1}\left(\mathbb{P}^{5}, \mathcal{I}_{X}(k)\right)=0$ for all $k \in \mathbb{Z}$.

Corollary 3.10. If $X$ is a smooth threefold in $\mathbb{P}^{5}$ of degree $d \leq 6$ and $X$ is cut out scheme-theoretically by three equations of degrees $d_{1} \geq d_{2} \geq d_{3}$, then $X$ is projectively normal.

Proof. Since $d_{3} \leq d_{2} \leq d_{1} \leq d-1$ ([3] , Corollary 4), $d_{1}+d_{2} \leq 10$. By Corollary 3.9. $X$ is projectively normal.

Example 3.11. Let $\mathcal{E}$ be a Horrocks-Mumford vector bundle of rank 2 with $c_{1}(\mathcal{E})=$

5 and $c_{2}(\mathcal{E})=10$. If $X$ is a non-minimal abelian surface of degree 15 in $\mathbb{P}^{4}$, then $X$ is cut out by three quintics, and we have the following locally free resolution ([2], $\S 7)$ :

$$
0 \rightarrow \mathcal{E}(-10) \rightarrow \bigoplus_{i=1}^{3} \mathcal{O}_{\mathbb{P}^{4}}(-5) \rightarrow \mathcal{I}_{X} \rightarrow 0
$$


Then, from the table of $\operatorname{dim} H^{i}(\mathcal{E}(q))$ in [12, page 74 , one can find that $H^{1}\left(\mathbb{P}^{4}, \mathcal{I}_{X}(k)\right)=0$ for $k \neq 5$ and $\operatorname{dim} H^{1}\left(\mathbb{P}^{4}, \mathcal{I}_{X}(5)\right)=2$. Note that $H^{2}\left(\mathbb{P}^{4}, \mathcal{I}_{X}(k)\right)$ $\neq 0$ for $0 \leq k \leq 3$. This gives the sharpness of Theorem 3.8

Remark 3.12. Hartshorne conjectures that every holomorphic rank 2 vector bundle over $\mathbb{P}^{n}, n \geq 7$, splits. This would be equivalent to the statement that every smooth subvariety $X \subset \mathbb{P}^{n}$ is a global complete intersection for $n \geq 7$. In fact until now no indecomposable rank 2 vector bundles over $\mathbb{P}^{n}, n \geq 5$, are known. If all the rank 2 vector bundles over $\mathbb{P}^{5}$ split, then all the Cohen-Macaulay quasi-complete intersections are projectively Cohen-Macaulay and almost complete intersections.

\section{ACKNOWLEDGEMENTS}

It is a pleasure to thank Professors S. Kwak and P. Kang for valuable discussions and encouragements. The author also thanks Professor M. Chang for helpful comments about Theorem 3.3 and the referee for pointing out [5] to me and for a careful reading of this paper.

\section{REFERENCES}

[1] D. Arapura, D. Jaffe, On Kodaira vanishing for singular varieties, Proc. Amer. Math. Soc. 105 (1989) 911-916. MR0952313 (89h:14013)

[2] A. Aure, W. Decker, K. Hulek, S. Popescu, K. Ranestad, Syzygies of abelian and bielliptic surfaces in $\mathbb{P}^{4}$, Internat. J. Math. 7 (1997) 849-919. MR1482969 (99a:14049)

[3] V. Beorchia, Ph. Ellia, On the equations defining quasi complete intersection space curves, Arch. Math. 70 (1998) 244-249. MR:1604080 (98k:14042)

[4] A. Bertram, L. Ein, R. Lazarsfeld, Vanishing theorems, a theorem of Severi, and the equations defining projective varieties, J. of Amer. Math. Soc. 4 (1991) 587-602. MR1092845 (92g:14014)

[5] H. Bresinsky, P. Schenzel, J. Stückrad, Quasi-complete intersection ideals of height 2, J. Pure Appl. Algebra 127 (1998) 137-145. MR1620704 (99d:13014)

[6] Y. Choi, S. Kwak, Remarks on the defining equations of smooth threefolds in $\mathbb{P}^{5}$, Geom. Dedicata 96 (2003) 151-159. MR.1956837(2003k:14052)

[7] W. Decker, S. Popescu, On surfaces in $\mathbb{P}^{4}$ and 3-folds in $\mathbb{P}^{5}$, Vector bundles in algebraic geometry (Durham, 1993), 69-100, London Math. Soc. Lecture Note Ser. 208 Cambridge Univ. Press, Cambridge, 1995. MR1338413 (96d:14046)

[8] L. Ein, R. Lazarsfeld, Syzygies and Koszul cohomology of smooth projective varieties of arbitrary dimension, Invent. Math. 111 (1993) 51-67. MR1193597 (93m:13006)

[9] G. Faltings, Ein Kriterium fur vollständig Durschnitte, Invent. Math. 62 (1981) 393-401. MR0604835 (82f:14050)

[10] D. Franco, L. Kleiman, T. Lascu, Gherardelli Linkage and Complete Intersections, Michigan Math. J. 48 (2000) 271-279. MR1786490(2002a:14057)

[11] M. Fiorentini, A. Lascu, A criterion for quasi complete intersections and related embedding questions, Ann. Univ. Ferrara 28 (1982) 153-166. MR0701894 (85b:14066)

[12] G. Horrocks, D. Mumford, Rank 2 vector bundle on $\mathbb{P}^{4}$ with 15,000 symmetries, Topology 12 (1973) 63-81. MR0382279(52:3164)

[13] S. Kwak, Castelnuovo-Mumford regularity for smooth threefolds in $\mathbb{P}^{5}$ and extremal examples, J. Reine Angew. Math. 509 (1999) 21-34. MR.1679165 (2000e:14064)

[14] N. Kumar, Monads on projective spaces, Manuscripta Math. 112 (2003) 183-189. MR2064915 (2005g:14083)

[15] J. Migliore, Introduction to liasion theory and deficiency modules, Birkhäuser, 1998. MR.1712469 (2000g:14058)

[16] D. Peskine, L. Szpiro, Liaison des variétés algébriques I, Invent. Math. 26 (1974) 271-302. MR0364271 (51:526)

Department of Mathematics, Korea Advanced Institute of Science and Technology, 373-1 Gusung-Dong Yusung-Gu, Daejeon, Korea

E-mail address: ychoi@math.kaist.ac.kr 